EPJ Web of Conferences 71, 00134 (2014)

DOI: 10.1051/epjconf/20147100134

(C) Owned by the authors, published by EDP Sciences, 2014

\title{
Effective degrees of freedom in QCD thermodynamics
}

\author{
L. Turko ${ }^{1, a}$, D. Blaschke ${ }^{1,2, b}$, D. Prorok ${ }^{1, c}$, and J. Berdermann $n^{3, d}$ \\ ${ }^{1}$ Instytut Fizyki Teoretycznej, Uniwersytet Wrocławski, Poland \\ ${ }^{2}$ Bogoliubov Laboratory for Theoretical Physics, JINR Dubna, Russia \\ ${ }^{3}$ German Aerospace Center, Institute of Communications and Navigation, Neustrelitz, Germany
}

\begin{abstract}
An effective model reproducing the equation of state of hadronic matter as obtained in recent lattice QCD simulations and from hadron resonance gas data is presented. The hadronic phase is described by means of an extended Mott-Hagedorn resonance gas while the QGP phase is described by the extended PNJL model. The dissociation of hadrons is obtained by including the state dependent hadron resonance width. The model gives a quantitative estimate for partial fractions of hadronic and partonic degrees of freedom above $T_{c}$.
\end{abstract}

\section{Introduction}

Simulations of lattice QCD (LQCD) are in practice the only reliable approach to QCD thermodynamics which covers the broad region of strongly interacting matter properties from the hadron gas at low temperatures to a deconfined quark gluon plasma phase at high temperatures. Recently, finite temperature LQCD simulations have overcome the difficulties of reaching the low physical light quark masses and approaching the continuum limit which makes this theoretical laboratory now a benchmark for modeling QCD under extreme conditions.

We are going to present a combined effective model reproducing the equation of state of hadronic matter as obtained in recent lattice QCD simulations [1,2]. The model should reproduce basic physical characteristics of processes encountered in dense hadronic matter, from the hot QCD phase through the critical temperature region till the lower temperature hadron resonance gas phase. In medium properties of hadrons are different from those in the vacuum. The very notion of the mass shell should be modified, as was postulated quite long ago [3]. The interaction becomes effectively nonlocal due to the Mott effect and hadrons eventually gradually dissolve into quarks and gluons in the high temperature phase. Then, with the increasing temperature, quark masses are less and less important although the massless Stefan-Boltzmann limit would be eventually reached only at extremely high temperature.

It has been shown that the equation of state derived from that time QCD lattice calculation [4] can be reproduced by a simple hadron gas resonance model. The rapid rise of the number of degrees of freedom in lattice QCD data around the critical temperature $T_{c} \sim 150-170 \mathrm{MeV}$, can be explained quantitatively by a resonance gas below the critical temperature $T_{c}[5,6]$.

\footnotetext{
ae-mail: turko@ift.uni.wroc.pl

be-mail: blaschke@ift.uni.wroc.pl

ce-mail: prorok@ift.uni.wroc.pl

de-mail: jens.berdermann@dlr.de
}

This is an Open Access article distributed under the terms of the Creative Commons Attribution License 2.0, which permits unrestricted use, distribution, and reproduction in any medium, provided the original work is properly cited. 
For higher temperatures the model is modified by introducing finite widths of heavy hadrons [7, 8] with a heuristic ansatz for the spectral function which reflects medium modifications of hadrons. This fits nicely the lattice data, also above $T_{c}$ as is shown in Fig. 1.

This Mott-Hagedorn type model $[9,10]$ has been constructed to fit nicely the lattice data, also above $T_{c}$ where it does so because it leaves light hadrons below a mass threshold of $m_{0}=1 \mathrm{GeV}$ unaffected. The description of the lattice data at high temperatures is accidental because the effective number of those degrees of freedom approximately coincides with that of quarks and gluons. The QGP presence in this region is formally simulated here by the smart choice of the mass cut-off parameter such that cut-off defined stable light hadrons provide the same number of degrees of freedom as partonic components of the QGP.

The considered model is here gradually refined to take into account those physical processes present in the full QCD treatment. The uniform treatment of all hadronic resonances, without artificial stability island, is reached by a state-dependent hadron resonance width $\Gamma_{i}(T)$ given by the inverse collision time scale in a resonance gas [11].

In order to remove this unphysical aspect of the otherwise appealing model one has to extend the spectral broadening also to the light hadrons and thus describe their disappearance due to the Mott effect while simultaneously the quark and gluon degrees of freedom appear at high temperatures due to chiral symmetry restoration and deconfinement.

In the present contribution we will report results obtained by introducing a unified treatment of all hadronic resonances with a state-dependent width $\Gamma_{i}(T)$ in accordance with the inverse hadronic collision time scale from a recent model for chemical freeze-out in a resonance gas [11]. The appearance of quark and gluon degrees of freedom is introduced by the Polyakov-loop improved Nambu-JonaLasinio (PNJL) model $[12,13]$. The model is further refined by adding perturbative corrections to $O\left(\alpha_{s}\right)$ for the high-momentum region above the three-momentum cutoff inherent in the PNJL model. One obtains eventually a good agreement with lattice QCD data, comparable with all important physical characteristics taken into account.

\section{Extended Mott-Hagedorn resonance gas}

\subsection{No quarks and gluons; hadronic spectral function with state-independent ansatz}

We introduce the width $\Gamma$ of a resonance in the statistical model through the spectral function

$$
A(M, m)=N_{M} \frac{\Gamma \cdot m}{\left(M^{2}-m^{2}\right)^{2}+\Gamma^{2} \cdot m^{2}},
$$

a Breit-Wigner distribution of virtual masses with a maximum at $M=m$ and the normalization factor

$$
N_{M}=\left[\int_{m_{0}^{2}}^{\infty} d\left(M^{2}\right) \frac{\Gamma \cdot m}{\left(M^{2}-m^{2}\right)^{2}+\Gamma^{2} \cdot m^{2}}\right]^{-1}=\frac{1}{\frac{\pi}{2}+\arctan \left(\frac{m^{2}-m_{0}^{2}}{\Gamma \cdot m}\right)} .
$$

The logarithm of the grand canonical partition function for hadrons and resonances can be written as

$$
\begin{aligned}
\log Z\left(T, V, \mu_{B}, \mu_{S}\right) & =V \sum_{i: m_{i}<m_{0}} g_{i} \delta_{i} \int \frac{d^{3} k}{(2 \pi)^{3}} \log \left(1+\delta_{i} e^{-\left(\sqrt{k^{2}+m_{i}^{2}}-\mu_{i}\right) / T}\right) \\
& +V \sum_{i: m_{i} \geq m_{0}} g_{i} \delta_{i} \int_{m_{0}^{2}}^{\infty} d\left(M^{2}\right) A\left(M, m_{i} \int \frac{d^{3} k}{(2 \pi)^{3}} \log \left(1+\delta_{i} e^{-\left(\sqrt{k^{2}+m_{i}^{2}}-\mu_{i}\right) / T}\right)\right.
\end{aligned}
$$


with the degeneracy $g_{i}$ and the chemical potential $\mu_{i}=B_{i} \cdot \mu_{B}+S_{i} \cdot \mu_{S}$ of hadron $i . \mu_{B}$ and $\mu_{S}$ are chemical potentials for baryon number and for strangeness respectively. For mesons, $\delta_{i}=-1$ and for baryons $\delta_{i}=1$.

And the model ansatz for the resonance width $\Gamma$ is given by [7]

$$
\Gamma(T)=C_{\Gamma}\left(\frac{m}{T_{H}}\right)^{N_{m}}\left(\frac{T}{T_{H}}\right)^{N_{T}} \exp \left(\frac{m}{T_{H}}\right),
$$

where $C_{\Gamma}=10^{-4} \mathrm{MeV}, N_{m}=2.5, N_{T}=6.5$ and the Hagedorn temperature $T_{H}=165 \mathrm{MeV}$.

The internal energy density of this model with zero resonance proper volume for given temperature $T$ and chemical potentials $\mu_{B}, \mu_{S}$ for strangeness, can be cast in the form

$$
\varepsilon\left(T, \mu_{B}, \mu_{S}\right)=\sum_{i: m_{i}<m_{0}} g_{i} \varepsilon_{i}\left(T, \mu_{i} ; m_{i}\right)+\sum_{i: m_{i} \geq m_{0}} g_{i} \int_{m_{0}^{2}}^{\infty} d\left(M^{2}\right) A\left(M, m_{i}\right) \varepsilon_{i}\left(T, \mu_{i} ; M\right)
$$

where $m_{0}=1 \mathrm{GeV}$ and the internal energy density per degree of freedom with a mass $M$ is

$$
\varepsilon_{i}\left(T, \mu_{i} ; M\right)=\int \frac{d^{3} k}{(2 \pi)^{3}} \frac{\sqrt{k^{2}+M^{2}}}{\exp \left(\frac{\sqrt{k^{2}+M^{2}}-\mu_{i}}{T}\right)+\delta_{i}},
$$

According to Eq. (1) the energy density of hadrons consists of the contribution of light hadrons for $m_{i}<m_{0}$ and the contribution of heavier hadrons smeared with the spectral function for $m_{i} \geq m_{0}$.

For simplicity, we assume $n_{S}=0$ for the strangeness number density and $n_{B}=0$ for the baryon number density. Then $\mu_{B}=0$ and $\mu_{S}=0$ always, so the temperature is the only significant statistical parameter here.

The pressure $(P)$ is obtained from the thermodynamic relation

$$
P=\frac{\partial(T \log Z)}{\partial V}
$$

which becomes in our case

$$
P=\frac{T}{V} \log Z
$$

The sound velocity squared for zeroth chemical potentials is given by

$$
c_{s}^{2}=\frac{\partial P}{\partial \varepsilon}
$$

In Fig. 1 we show the results for the thermodynamic quantities (pressure, energy density and squared speed of sound) of the MHRG model at this stage. The nice correspondence with results from lattice QCD is not accidental for the temperature region $T \sim T_{c} \sim 200 \mathrm{MeV}$, where has been shown in [1] that a hadron resonance gas perfectly describes the lattice QCD data. For $T>T_{c}$ the broadening of the spectral function (1) which affects at this stage of the model only the hadronic resonances with $m>m_{0}$ leads to the vanishing of their contribution at about $2 T_{c}$ while the light hadrons with masses $m<m_{0}$ are not affected and gradually reach the Stefan- Boltzmann (SB) limit determined by their number of degrees of freedom. As has been noted in [14], this number $\left(\sum_{i=\pi, K, \eta, f_{0}, \rho, \omega, K^{*}, \eta^{\prime}, f_{0}, a_{0}} g_{i}=\right.$ $3+4+1+1+9+3+6+1+1+3=32$ ) accidentally (or by duality arguments) coincides with that of the quarks and gluons $\left(\sum_{i=q, g} g_{i}=7 / 8 * N_{c} * N_{f} * N_{s} * 2+\left(N_{c}^{2}-1\right) * 2=31.5\right)$ for $N_{c}=N_{f}=3$. 
Therefore, imposing that all mesons lighter than $m_{0}=1 \mathrm{GeV}$ are stable provides us with a SB limit at high temperatures which fakes that of quarks and gluons in the case for three flavors.

Although providing us with an excellent fit of the lattice data, the high-temperature phase of this model is unphysical since it ignores the Mott effect for light hadrons. Due to the chiral phase transition at $T_{c}$, the quarks loose their mass and therefore the threshold of the continuum of quark-antiquark scattering states is lowered. At the same time the light meson masses, however, remain almost unaffected by the increase in the temperature of the system. Consequently, they merge the continuum and become unbound - their spectral function changes from a delta-function (on-shell bound states) to a Breit-Wigner-type (off-shell, resonant scattering states). This phenomenon is the hadronic analogue $[15,16]$ of the Mott-Anderson transition for electrons in solid state physics (insulator-conductor transition).

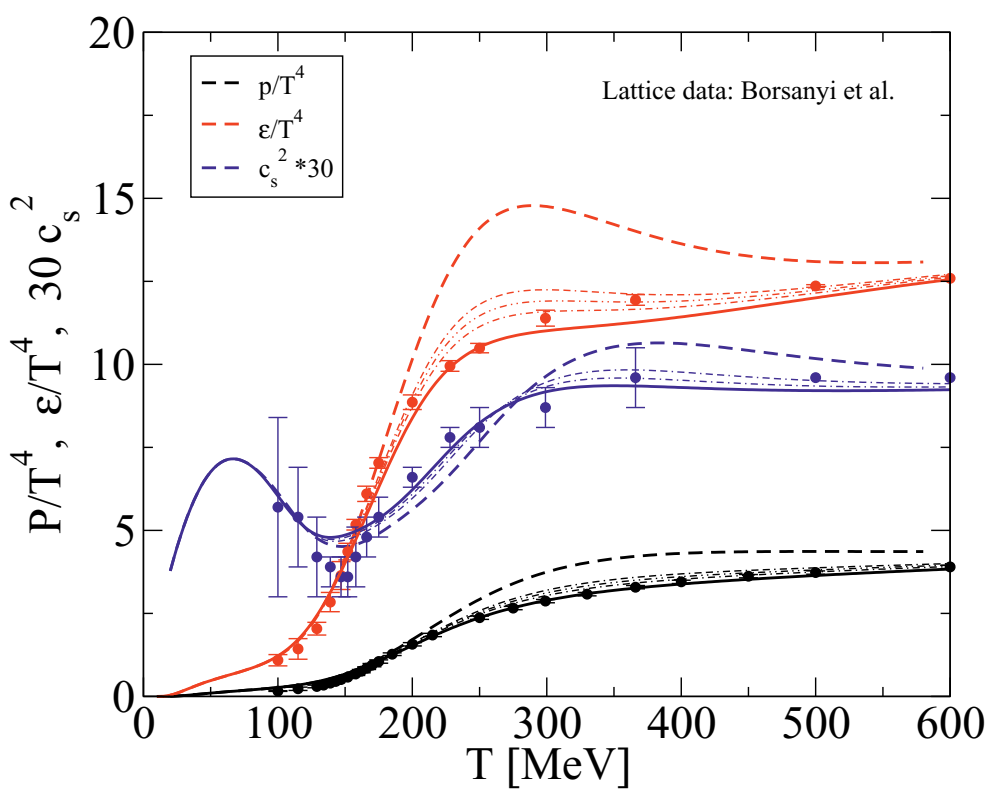

Figure 1. (Color online) Thermodynamic quantities for the old Mott-Hagedorn Resonance Gas model [7]. Different line styles correspond to different values for the parameter $N_{m}$ in the range from $N_{m}=2.5$ (dashed line) to $N_{m}=3.0$ (solid line). Lattice QCD data are from Ref. [1].

It has been first introduced for the hadronic-to-quark-matter transition in [17]. Later, within the NJL model, a microscopic approach to the thermodynamics of the Mott dissociation of mesons in quark matter has been given in the form of a generalized Beth-Uhlenbeck equation of state [18], see also [19]. Recently, a detailed treatment of the Mott dissociation of two-quark correlations [16] and in particular of pions [20-22] was give within the PNJL model as well as its nonlocal generalization [23].

\subsection{Hadronic spectral function with state-dependent ansatz}

As a microscopic treatment of the Mott effect for all resonances is presently out of reach, we introduce an ansatz for a state-dependent hadron resonance width $\Gamma_{i}(T)$ given by the inverse collision time scale 
recently suggested within an approach to the chemical freeze-out and chiral condensate in a resonance gas [11]

$$
\Gamma_{i}(T)=\tau_{\mathrm{coll}, \mathrm{i}}^{-1}(T)=\sum_{j} \lambda\left\langle r_{i}^{2}\right\rangle_{T}\left\langle r_{j}^{2}\right\rangle_{T} n_{j}(T),
$$

which is based on a binary collision approximation and relaxation time ansatz using for the in-medium hadron-hadron cross sections the geometrical Povh-Hüfner law [24]. In Eq. (8) the coefficient $\lambda$ is a free parameter, $n_{j}(T)$ is the partial density of the hadron $j$ and the mean squared radii of hadrons $\left\langle r_{i}^{2}\right\rangle_{T}$ obtain in the medium a temperature dependence which is governed by the (partial) restoration of chiral symmetry. For the pion this was quantitatively studied within the NJL model [25] and it was shown that close to the Mott transition the pion radius is well approximated by

$$
r_{\pi}^{2}(T)=\frac{3}{4 \pi^{2}} f_{\pi}^{-2}(T)=\frac{3 M_{\pi}^{2}}{4 \pi^{2} m_{q}}\left|\langle\bar{q} q\rangle_{T}\right|^{-1} .
$$

Here the Gell-Mann-Oakes-Renner relation has been used and the pion mass shall be assumed chirally protected and thus temperature independent.

For the nucleon, we shall assume the radius to consist of two components, a medium independent hard core radius $r_{0}$ and a pion cloud contribution $r_{N}^{2}(T)=r_{0}^{2}+r_{\pi}^{2}(T)$, where from the vacuum values $r_{\pi}=0.59 \mathrm{fm}$ and $r_{N}=0.74 \mathrm{fm}$ one gets $r_{0}=0.45 \mathrm{fm}$. A key point of our approach is that the temperature dependent hadronic radii shall diverge when hadron dissociation (Mott effect) sets in, driven basically by the restoration of chiral symmetry. As a consequence, in the vicinity of the chiral restoration temperature all meson radii shall behave like that of the pion and all baryon radii like that of the nucleon.

The resulting energy density behaviour is shown in Fig. 2. This part of the model we call Mott-

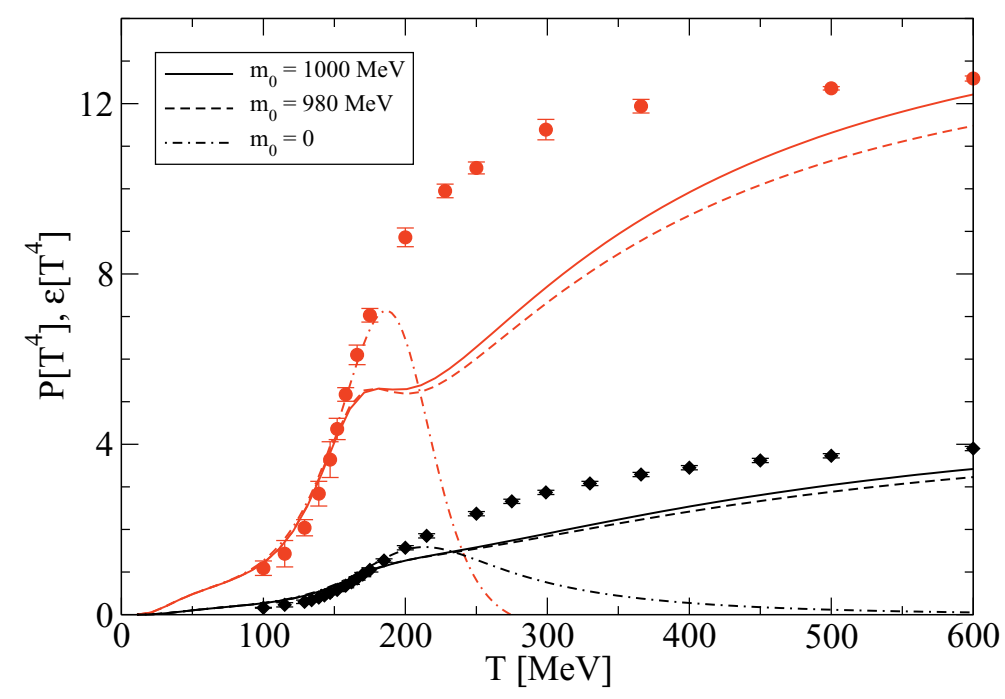

Figure 2. (Color online) Energy density (red lines and symbols) and pressure (black lines and symbols) for the state-dependent width model of Eq. (8) and three values of the mass threshold $m_{0}: 1 \mathrm{GeV}$ (solid lines), $980 \mathrm{MeV}$ (dashed lines), 0 (dash-dotted lines). Lattice QCD data are from Ref. [1].

Hagedorn-Resonance-Gas (MHRG). When all hadrons are gone at $T \sim 250 \mathrm{MeV}$, we are clearly missing degrees of freedom! 


\section{Quarks, gluons and hadron resonances above $T_{c}$}

We improve the PNJL model over its standard versions $[12,13]$ by adding perturbative corrections in $O\left(\alpha_{s}\right)$ for the high-momentum region above the three-momentum cutoff $\Lambda$. In the second step, the MHRG part is replaced by its final form, using the state-dependent spectral function for the description of the Mott dissociation of all hadron resonances above the chiral transition. The total pressure obtains the form

$$
P(T)=P_{\mathrm{MHRG}}(T)+P_{\mathrm{PNJ}, \mathrm{MF}}(T)+P_{2}(T) .
$$

where $P_{\mathrm{MHRG}}(T)$ stands for the pressure of the MHRG model, accounting for the dissociation of hadrons in hot dense, matter.

The $O\left(\alpha_{s}\right)$ corrections can be split in quark and gluon contributions

$$
P_{2}(T)=P_{2}^{\text {quark }}(T)+P_{2}^{\text {gluon }}(T),
$$

where $P_{2}^{\text {quark }}$ stands for the quark contribution and $P_{2}^{\text {gluon }}$ contains the ghost and gluon contributions. The total perturbative QCD correction to $O\left(\alpha_{s}\right)$ is

$$
P_{2}=-\frac{8}{\pi} \alpha_{s} T^{4}\left(I_{\Lambda}^{+}+\frac{3}{\pi^{2}}\left(\left(I_{\Lambda}^{+}\right)^{2}+\left(I_{\Lambda}^{-}\right)^{2}\right)\right)
$$

where $I_{\Lambda}^{ \pm}=\int_{\Lambda / T}^{\infty} \frac{\mathrm{d} x x}{\mathrm{e}^{x} \pm 1}$. The corresponding contribution to the energy density is given in standard way from the thermodynamic relation

$$
\varepsilon+P=T \cdot s=T \cdot \frac{\partial P}{\partial T},
$$

We will now include an effective description of the dissociation of hadrons due to the Mott effect into the hadron resonance gas model by including the state dependent hadron resonance width (8) into the definition of the HRG pressure

$$
P_{\mathrm{MHRG}}(T)=\sum_{i} \delta_{i} d_{i} \int \frac{d^{3} p}{(2 \pi)^{3}} d M A_{i}(M) T \ln \left(1+\delta_{i} \mathrm{e}^{-\sqrt{p^{2}+M^{2}} / T}\right) .
$$

From the pressure as a thermodynamic potential all relevant thermodynamical functions can be obtained. Combining the $\alpha_{s}$ corrected meanfield PNJL model for the quark-gluon subsystem with the MHRG description of the hadronic resonances we obtain the results shown in the Fig. 3 where the resulting partial contributions in comparison with lattice QCD data from Ref. [1] are shown.

We see that the lattice QCD thermodynamics is in full accordance with a hadron resonance gas up to a temperature of $\sim 170 \mathrm{MeV}$ which corresponds to the pseudocritical temperature of the chiral phase transition. The lattice data saturate below the Stefan-Boltzmann limit of an ideal quark-gluon gas at high temperatures. The PNJL model, however, attains this limit by construction. The deviation is to good accuracy described by perturbative corrections to $O\left(\alpha_{s}\right)$ which vanish at low temperatures due to an infrared cutoff procedure. The transition region $170 \leq T[\mathrm{MeV}] \leq 250$ is described by the MHRG model, resulting in a decreasing HRG pressure which vanishes at $T \sim 250 \mathrm{MeV}$.

We have presented two stages of an effective model description of QCD thermodynamics at finite temperatures which properly accounts for the fact that in the QCD transition region it is dominated by a tower of hadronic resonances. To this end we have further developed a generalization of the Hagedorn resonance gas thermodynamics which includes the finite lifetime of hadronic resonances in a hot and dense medium by a model ansatz for a temperature- and mass dependent spectral function. 

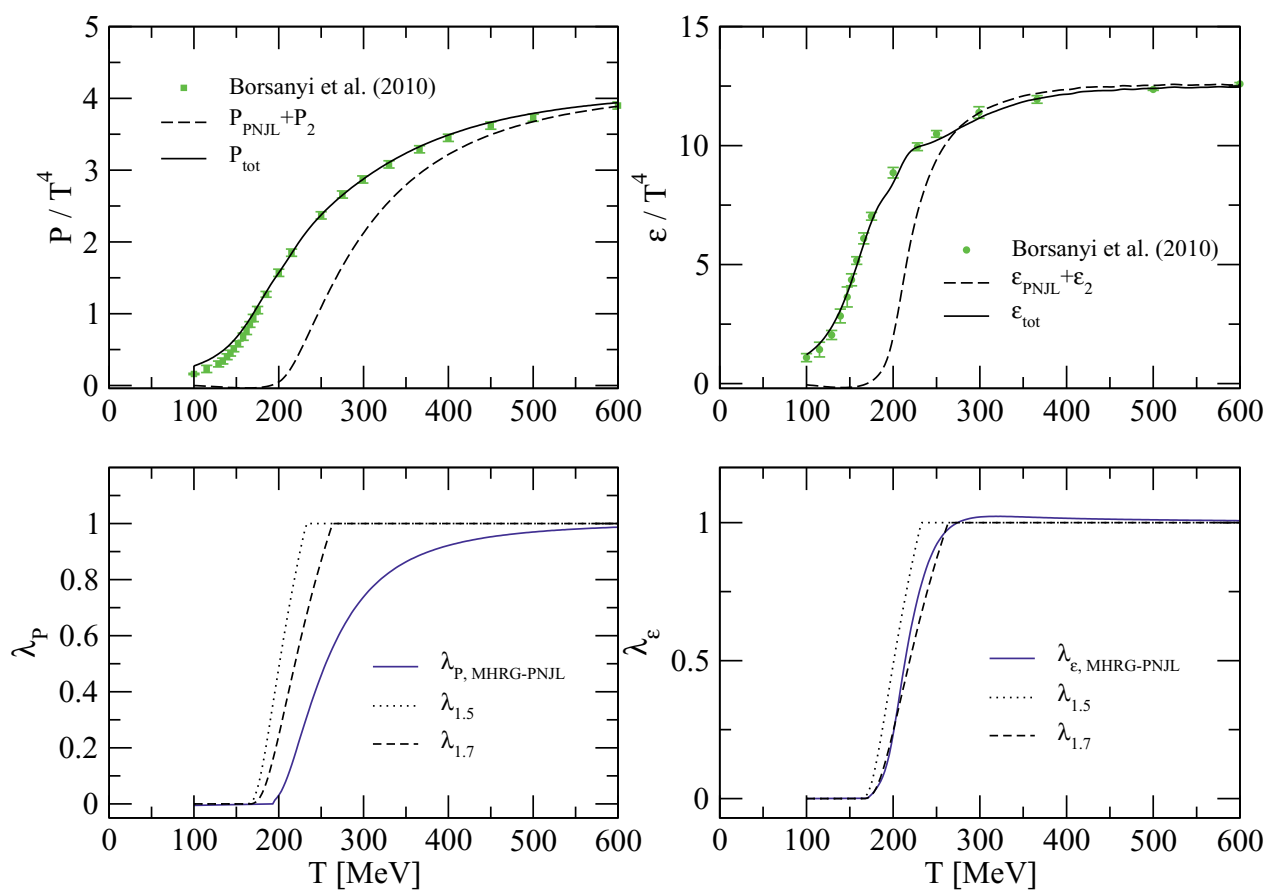

Figure 3. (Color online) Thermodynamic quantities (pressure - left panels; energy density - right panels) for the new Mott-Hagedorn Resonance Gas where quark-gluon plasma contributions are described within the PNJL model including $\alpha_{s}$ corrections (dashed lines). The total quantites are shown by full lines and compared to lattice QCD data [1] in the upper panels. In the lower panels we show the fraction of partonic pressure (lower left panel) and the fraction of partonic energy density (lower right panel) by the solid blue lines, resp. Also shown in these lower panels is the fraction of partonic degrees of freedom $\lambda$, as introduced in Ref. [27].

\section{Conclusion and outlook}

After having presented the MHRG-PNJL model with the state-dependent spectral function approach we show the summary of the thermodynamic quantities in Fig. 3. We have presented two stages of an effective model description of QCD thermodynamics at finite temperatures which properly accounts for the fact that in the QCD transition region it is dominated by a tower of hadronic resonances. In the first of the two stages of the developments we presented here, we have used the fact that the number of low-lying mesonic degrees of freedom with masses below $\sim 1 \mathrm{GeV}$ approximately equals that of the thermodynamic degrees of freedom of a gas of quark and gluons. In the second one we have further developed a generalization of the Hagedorn resonance gas thermodynamics which includes the finite lifetime of heavy resonances in a hot and dense medium by a model ansatz for a temperature- and mass dependent spectral function which is motivated by a model for the collision time successfully applied in the kinetic description of chemical freeze-out from a hadron resonance gas.

The presented formalism allows for the analysis of relative contributions originating from partonic and hadronic degrees of freedom. Those latter ones, still present even for temperatures higher than the critical one [26] lead to clear phenomenological effects. In particular, they significantly influence the behavior of heavy quark observables in the hot QCD phase above $T_{c}$. The recent analysis of this effect [27] was based on rather arbitrary assumptions concerning hadronic degrees of freedom 
at higher temperatures. By comparing improved PNJL and MHRG contributions to the pressure and energy density, as presented in Fig. 3, we were able to extract the fraction of pressure or energy density carried by partonic degrees of freedom - without going beyond our model assumptions. This opens interesting new applications of the approach presented here to the phenomenology of ultra-relativistic heavy-ion collisions.

\section{Acknowledgment}

This work was supported in part by the Polish National Science Center (NCN) under contract No. N N202 052340 and the "Maestro" programme UMO-2011/02/A/ST2/00306 (D.B.).

\section{References}

[1] S. Borsanyi et al., JHEP 1011, 077 (2010).

[2] A. Bazavov et al., Phys. Rev. D 80, 014504 (2009).

[3] F. Karsch, K. Redlich and L. Turko, Z. Phys. C 60, 519 (1993).

[4] F. Karsch, Lect. Notes Phys. 583, 209 (2002); [hep-lat/0106019].

[5] F. Karsch, K. Redlich and A. Tawfik, Eur. Phys. J. C 29, 549 (2003).

[6] C. Ratti et al. [Wuppertal-Budapest Collaboration], Nucl. Phys. A 855, 253 (2011).

[7] D. B. Blaschke, K. A. Bugaev, Fizika B 13, 491 (2004).

[8] D. B. Blaschke, K. A. Bugaev, Phys. Part. Nucl. Lett. 2, 305 (2005).

[9] L. Turko, D. Blaschke, D. Prorok and J. Berdermann, Acta Phys. Polon. Supp. 5, 485 (2012).

[10] L. Turko, D. Blaschke, D. Prorok and J. Berdermann, J. Phys. Conf. Ser. 455, 012056 (2013).

[11] D. B. Blaschke et al., Phys. Part. Nucl. Lett. 8, 811 (2011).

[12] K. Fukushima, Phys. Lett. B 591, 277 (2004).

[13] C. Ratti, M. A. Thaler, W. Weise, Phys. Rev. D 73, 014019 (2006).

[14] G. E. Brown, H. A. Bethe, P. M. Pizzochero, Phys. Lett. B 263, 337 (1991).

[15] D. Zablocki, D. Blaschke and G. Röpke, Metal-to-Nonmetal Transitions, Springer Series in Materials Science, 132, 161 (2010).

[16] D. Blaschke et al., arXiv:1305.3907 [hep-ph].

[17] D. Blaschke et al., Phys. Lett. B 151, 439 (1985).

[18] J. Hüfner et al., Annals Phys. 234, 225 (1994).

[19] A. E. Radzhabov et al., Phys. Rev. D 83, 116004 (2011).

[20] A. Wergieluk et al., Phys. Part. Nucl. Lett. 10, 660 (2013).

[21] K. Yamazaki and T. Matsui, Nucl. Phys. A 913, 19 (2013).

[22] A. Dubinin et al., arXiv:1312.0559 [hep-ph].

[23] S. Benić and D. Blaschke, Acta Phys. Polon. Supp. 6, no. 3, 947 (2013).

[24] B. Povh, J. Hüfner, Phys. Lett. B 245, 653 (1990).

[25] H. J. Hippe and S. P. Klevansky, Phys. Rev. C 52, 2172 (1995).

[26] C. Ratti, R. Bellwied, M. Cristoforetti and M. Barbaro, Phys. Rev. D 85, 014004 (2012).

[27] M. Nahrgang, J. Aichelin, P. B. Gossiaux and K. Werner, Phys. Rev. C 89, 014905 (2014). 